(C) 2010 IEEE. Personal use of this material is permitted. Permission from IEEE must be obtained for all other uses, in any current or future media, including reprinting/republishing this material for advertising or promotional purposes, creating new collective works, for resale or redistribution to servers or lists, or reuse of any copyrighted component of this work in other works. 


\title{
Multi-class Classification for Semantic Labeling of Places
}

\author{
Lei Shi, Sarath Kodagoda, and Gamini Dissanayake, Member, IEEE \\ ARC Centre of Excellence for Autonomous Systems (CAS) \\ The University of Technology \\ Sydney, Australia
}

\begin{abstract}
Human robot interaction is an emerging area of research, where human understandable robotic representations can play a major role. Knowledge of semantic labels of places can be used to effectively communicate with people and to develop efficient navigation solutions in complex environments. In this paper, we propose a new approach that enables a robot to learn and classify observations in an indoor environment using a labeled semantic grid map, which is similar to an Occupancy Grid like representation. Classification of the places based on data collected by laser range finder (LRF) is achieved through a machine learning approach, which implements logistic regression as a multi-class classifier. The classifier output is probabilistically fused using independent opinion pool strategy. Appealing experimental results are presented based on a data set gathered in various indoor scenarios.
\end{abstract}

Keywords - semantic labeling of places, logistic regression, data fusion, independent opinion pool, semantic grid map

\section{INTRODUCTION}

Detection, classification and representation of semantic labels of places are non trivial yet important. They enable a robot to describe the environment intelligently and concisely, which may lead to a common representation of high level information that can be effectively and efficiently shared between humans and robots. As a result, the robots may be able to effectively communicate with humans to carry out complex or interactive tasks in a shared space.

Most popular sensors that have been used in semantic labeling of places are cameras and 2D laser range finders (LRF). Cameras provide large amount of information as colour, texture and 3D structure (in case of stereo), which are beneficial to classification. However, vision data is vulnerable to noise and lighting conditions, requires calibration, and increases the processing overhead. In contrast, 2D laser range data is attributed with low processing complexity, although it does not reflect as much information as a camera. Irrespective of their pros and cons, either of the sensors can be used for semantic labeling of places. For example, [1] and [5] are based on vision sensors, [6] and [7] are based on laser range finder data. Semantic labelling based on multi-sensory approach can be found in [14]. However, our belief is that the potential of the laser range finders has not yet been well exploited. Hence, in this paper, our aim is to semantically classify places based only on two dimensional (2D) laser range/bearing data. Following paragraph describes the most relevant literature.
Buschka et al. [2] proposed a rectangular-fit algorithm to incrementally extract room-like topological nodes and to segment the space into room and corridor regions. Poncela et al. [3] adopted Principal Component Analysis to classify the environment perceived by LRF into walls and doors. Both approaches rely on the invariant parameters of a certain space, such as width and length. Tapus et al. [4] proposed a Bayesian approach for topology recognition and door detection which considers complicated topological details. Mozos et al. [6] extracted variety of simple features from laser range data and made use of AdaBoost classifier to classify environments into rooms, corridors, doorways and halls. In a similar approach, Sousa et al. [7] classified places using Support Vector Machines (SVM). In both cases, only the positions of the robot rather than the obstacle points were labeled.

In this paper, we firstly select three dominant features and perform the classification task using logistic regression as multi-class classifier. The classification result can be used to label either the observer's positions or the obstacle points. Then we adopt the independent opinion pool approach to fuse the probabilities assigned to certain obstacle points perceived in different observations, resulting in a semantic grid map. Then, uncertain points were removed to further improve the classification accuracy.

The rest of this paper is arranged as follows. Section II discusses the details of the classification algorithm. Data fusion approach and map building issues are described in Section III. Section IV defines the inliers and outliers in every single laser scan and discusses the outlier removal approaches to increase the overall labeling accuracy. In Section V, experimental results are presented and Section VI concludes the paper.

\section{CLASSIFICATION}

Typically, a learning problem can be described by three elements, which are task, performance measure and training experience [16]. In this application, task is to label the environment perceived in an observation as belonging to a certain semantic label; performance measure is the accuracy of the judgment; and training experience is a sequence of observations with known semantic labels. This section provides a discussion on application, feature selection and classification strategy. 


\section{A. Semantic Labels}

In a typical university indoor environment most commonly observed semantic labels are "office rooms", "lecture rooms", "corridors" and "doorways". Doorways can be considered as a transition between one label type to the other and hence in this paper, we only label "office rooms", "lecture rooms" and "corridors" in a given environment.

\section{B. Feature Selection}

In supervised machine learning problems, feature selection is of significant importance because it directly affects the generalization ability, overhead and overfitting issues of the system. As is widely accepted in some applications that a small subset of features is sufficient to approximate the target concept well [10], finding the dominant features becomes a key issue in most classification problems.

A two dimensional LRF mounted on a mobile robot scanning in a horizontal plane provides range/bearing data to close-by objects. Due to the present of various furniture and other objects in the environment, the laser range/bearing data cannot explicitly capture the complete boarder of a particular label type. Because of this, laser scans taken at various poses in a particular label type can provide significantly different local features. However, gross features could be the same. As an example, laser range/bearing data corresponding to a corridor has a long rectangular shape when plotted in a Cartesian coordinate, whilst an office room shows a short rectangular shape.

There are various features of LRF data that have been utilized in the literature for semantic labeling of places. Mozos et al. [6][15] derived two sets of simple features from raw laser range/bearing data. One set is extracted from raw range data and the other is extracted from polygonal approximation of the observed area. Total number of 150 single-valued features was derived from 22 kinds of features considering different thresholds. Similarly, Sousa et al. [7] adopted 14 single-valued features.

We extracted similar features and evaluated all of them and some combinations using L2-regularized logistic regression as a multi-class classifier. Results show a high classification accuracy (giving due regards to computational overhead) with three dominant features out of the 150 features used in [6]. Therefore, the three features, which are described in detail in section V.B, are used as dominant features in this application.

\section{Logistic Regression As Classifier}

As stated in section I, some successful classifiers used in semantic labeling of places are AdaBoost [12] and Support Vector Machines (SVM) [13]. AdaBoost constructs a strong classifier as linear combination of many weak classifiers, and SVM performs classification in a high-dimensional feature space and has the advantage of dealing with small training samples. However, in this paper, we adopt logistic regression as classifier because it is able to provide outputs in a probabilistic form, which enables us to integrate these outputs in a map building process.

Logistic Regression is a method to learn functions of the form $P(y \mid \vec{x})$, where $y$ is discrete-valued random variable and $\vec{x}$ is any vector containing discrete or continuous variables [8]. It assumes a parametric form of the distribution $P(y \mid \vec{x})$ while directly estimating its parameters from the training data [8].

For binary classification, given data $\vec{x}$ and weights $\vec{w}$, the parametric model is:

$$
P(y= \pm 1 \mid \vec{x} ; \vec{w})=\frac{1}{1+\exp \left(-y \cdot \vec{w}^{T} \cdot \vec{x}\right)}
$$

Let the training samples be $\left(y_{i}, \vec{x}_{i}\right)$, where $\vec{x}_{i}$ is the feature set and $y_{i} \in\{+1,-1\}$ is the label of a certain training sample, then the training task is to minimize the negative loglikelihood,

$$
\min _{\vec{w}}\left(\sum_{i=1}^{m} \log \left(1+\exp \left(-y_{i} \cdot \vec{w}^{T} \cdot \vec{x}_{i}\right)\right)\right)
$$

However, for this algorithm, over fitting becomes a potential risk especially when data is with high dimension and training data is sparse [8]. Therefore, regularization which encourages the fitted parameters to be small is introduced to reduce over fitting [10]. Algorithms, such as L2 regularization, which encourages the sum of squares of the parameters to be small [10] are commonly used for this purpose.

L2-regularized algorithm solves the following problem:

$$
\min _{\vec{w}}\left(\frac{1}{2} \vec{w}^{T} \vec{w}+C \sum_{i=1}^{m} \log \left(1+\exp \left(-y_{i} \cdot \vec{w}^{T} \cdot \vec{x}_{i}\right)\right)\right)
$$

where $C>0$ is a penalty parameter.

Although logistic regression is originally a binary classifier, it has proven to be extended to deal with multi-class classification by applying strategies such as one-against-all, one-against-one etc [11]. In this paper, L2-regularized logistic regression is employed as a multi-class classifier.

\section{DATA FUSION}

\section{A. Independent Opinion Pool}

In the area of data fusion, there are many methods to fuse outputs in the form of either a likelihood $P(z \mid x)$ or an opinion $P(x \mid z)$, where $z$ is an observation and $x$ is a state of the target. Three common approaches to combine these probabilistic evidences are: linear opinion pool, independent opinion pool and independent likelihood pool [17][18].

In this application, observations are taken independently from a set of unique positions of the robot, which leads us to make use of the independent opinion pool approach to probabilistically update a semantic grid map of the robot's environment.

The independent opinion pool method can be described as follows: 


$$
P\left(x \mid z_{1}, \ldots, z_{n}\right)=\alpha \cdot \prod_{i}^{n} P\left(x \mid z_{i}\right)
$$

where $z_{i}$ is the $i^{\text {th }}$ observation of a particular grid point, $x$ is the state (semantic label) of that grid point, and $\alpha$ is a normalizing factor.

Equation (4) can be rewritten in a recursive format as follows:

$$
P\left(x \mid z_{1}, \ldots, z_{n}\right) \propto P\left(x \mid z_{1}, \ldots, z_{n-1}\right) \cdot P\left(x \mid z_{n}\right)
$$

Moreover, in our application, there are three possible states (semantic label) of grid points as stated in section II.A and they are exclusive. That is, the posterior probabilities of the point belonging to a certain state sums up to one.

\section{B. Semantic Grid Map Building}

By using equation (5) and the exclusive nature of three possible states, semantic grids can be updated as independent observations accumulatively.

The semantic grid map is analogous to its counterpart, occupancy grid map [19]. In occupancy grid map, grid points are described by the probabilities of being occupied, while in semantic grid map grid points are described by the probabilities of belonging to certain semantic label.

\section{LASER DATA PREPROCESSING}

The semantic map which is built using the independent opinion pool can have large number of misclassifications because the LRF sees through opened doors making part of range/bearing data to be belonging to adjacent label types. Therefore, it is proposed to reduce the misclassification rate by a further processing step.

\section{A. Inliers and Outliers}

Here, we define inliers as the laser range/bearing data in a particular scan belonging to a specific semantic label, and define outliers as the remainder of the data.

For example, if an observation taken in an office room with an open door contains obstacle points both in the same office room and in a corridor. Then the former are called inliers and the latter are called outliers. If inliers and outliers can be discriminated, then the probabilistic properties of the detected semantic grid points can be updated selectively, which leads to a higher labeling accuracy.

\section{B. Rectangle Filter}

One approach to discriminate inliers and outliers is by finding doors directly. Many techniques for door detection can be found in the literature [20][21]. However, it is erroneous to detect doors by matching fixed key elements like door width, and the result will be affected by the opening states of doors. Furthermore, the presence of furniture in most indoor scenarios gives rise to door-like openings.

We suggest to distinguish inliers and outliers using a rectangle filter, which is based on the intuition that most of the indoor spaces can be represented by a rectangle. Here we propose a hypothesis that most of the inliers lie within a rectangle and the area of the rectangle is restricted by the area of polygonal approximation of all LRF data points (both inliers and outliners). The hypothesis is based on the prior knowledge that most of engineered closed spaces can be represented by rectangles, and the number of inliers contributes mostly to a certain place class rather than outliers.

The hypothesis has its limitations in complex environments such as corridor intersections due to shape complexity. However, simulations show that it produces good results in most indoor scenarios.

The rectangle filter algorithm is implemented as follows:

- Find the main directions of a laser scan by line features

- Find all possible rectangles of the same area as that of a laser scan polygon, and align along the main directions.

- Among these rectangles, choose the one which contains the maximum number of laser data points (see Fig. 1.)

Once the appropriate rectangle is chosen, inliers are chosen to be positioned within the boundary and the rest of the data are considered to be outliers. This algorithm provides a conservative inlier prediction mechanism.
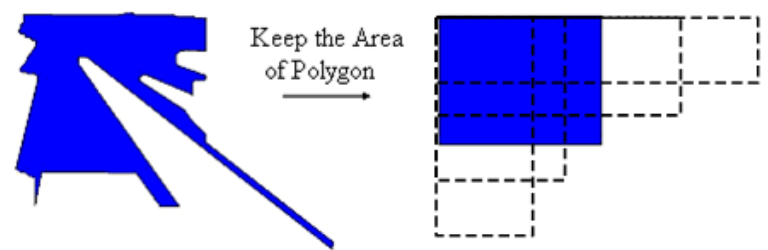

Fig. 1. Basic concept of the rectangle filter: Left figure shows the area enclosed by the polygon. Right figure shows some predicted rectangles (in dotted lines) and the finally decided rectangle (filled with blue)

\section{Selective Data Fusion}

By discriminating the inliers and outliers using rectangle filter, the properties of obstacle points perceived in a single observation can be updated selectively in the accumulatively labeling process described in section III.B.

Probabilities of semantic labels of inliers can be updated as usual using independent opinion pool method. However, due to the unknown nature of outlier labels, the semantic probabilities related to them are kept unchanged. Discarding unknown labels leads to higher classification accuracy.

\section{RESULTS}

\section{A. The Environment and the Data Set}

There are three data sets used in this study.

Data set DS1: This includes a data set published by Oscar [9]. It contains both raw laser range/bearing data and associated features for a robot operating in an office like environment, which consists of a corridor and several rooms of different sizes. The robot is equipped with a front and a rear 2D LRFs providing 360 degrees field of view. Training samples and testing samples are given from different parts of the environment. 
Data set DS2: A robot operating in an indoor environment (blueprint: Level 6, Building 2 of the University of the University of Technology, Sydney) is used. The space is consisted of 3 long corridors, 3 lecture rooms 15 office rooms of different shapes. The lecture rooms have tables and chairs whilst the office rooms have various furniture (see Fig. 2). The robot is equipped with two LRFs mounted back to back capturing 360 degrees scan in a particular time step. The doors are randomly opened (opened, partly opened, etc).

Data set DS3: The same operating environment, sensors and the robot are used. However, the doors are kept in fully opened status.

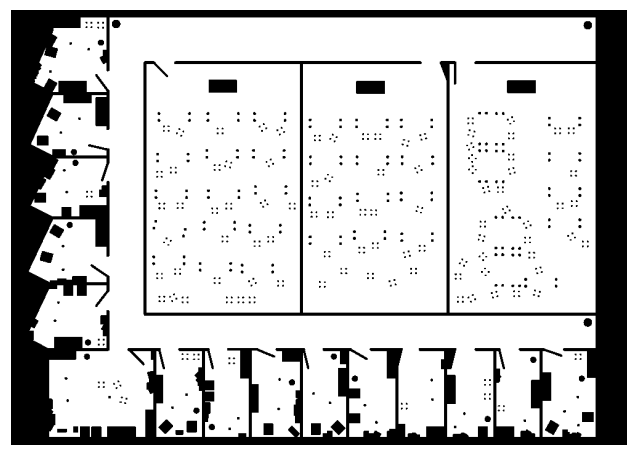

Fig. 2. Map of the simulated environment. The status of doors is random. In this map, there are 15 office rooms along the left and bottom edges and 3 lecture rooms in the middle. Office rooms and lecture rooms are separated by a long corridor which can be divided into 3 parts.

\section{B. Feature Selection}

In this experiment, data set DS1, which is a public data set with abundant candidate features, is employed to analyze feature selection issue. As mentioned before (Section II.B), Mozos et al. [6][15] derived two sets of features which are from raw range data (called B series here) and from polygonal approximation of the observed area (called $\mathrm{P}$ series here). In regard to the definition of these features, please refer to [6][7] [15].

The training and testing data consist of 52465 and 39050 samples respectively, and 150 features corresponding to every sample. A multi-class L2-regularized logistic regression classifier is employed to classify the perceived environment in observations into 3 classes based on different combinations of features. By testing and comparing many different feature combinations, the shortlisted feature combinations and their performances are listed in TABLE I.

TABLE I

PERFORMANCE OF DIFFERENT FEATURE COMBINATIONS

\begin{tabular}{|l|c|c|}
\hline \multicolumn{1}{|c|}{ Feature Combination } & $\begin{array}{c}\text { Testing } \\
\text { Error }\end{array}$ & $\begin{array}{c}\text { Testing } \\
\text { Time }\end{array}$ \\
\hline All 150 features & $1.97 \%$ & $6.44 \mathrm{~s}$ \\
\hline All 21 single-valued features & $2.09 \%$ & $1.07 \mathrm{~s}$ \\
\hline All 11 single-valued P series & $2.40 \%$ & $0.52 \mathrm{~s}$ \\
\hline All 10 single-valued B series & $2.57 \%$ & $0.53 \mathrm{~s}$ \\
\hline 3 selected features & $2.12 \%$ & $0.26 \mathrm{~s}$ \\
\hline
\end{tabular}

In TABLE I, the 3 selected features derived from a single observation are:
- The standard deviation of the difference between the lengths of consecutive ranges

- The standard deviation of ranges

- The area of polygonal approximation

TABLE I indicates that feature selection is a tradeoff between accuracy and overhead (or complexity). With all 150 features, the algorithm has the best classification accuracy however with a substantial computational burden. On the other hand 3 selected features provided reasonable accuracy with the lowest computational burden. Therefore, the 3 selected features are adopted in the following learning algorithm.

\section{Classification Results}

In this experiment, data set DS2 is used to classify observer's position belonging to various semantic labels based on gross LRF data. For this purpose, 2957 laser range scans have been used as the training samples (as shown in Fig. 3 (a)), and another 2956 scans have been used as the testing samples.

Classification is carried out using L2-regularized logistic regression as a multi-class classifier and the output is in the form of probability estimation. The performance of the classifier is shown in TABLE II, and visualized in Fig. 3 (b). It can be seen that the testing error of office rooms is very low whilst that of lecture rooms is the highest. This is due to the presence of various furniture, which occludes most parts of the laser scans and produces office room like features. Testing error of corridors is mainly due to the opened doors.

TABLE II

PERFORMANCE OF CLASSIFIER ON RANDOMLY OPENED DOORS SCENARIO

\begin{tabular}{|l|c|}
\hline \multicolumn{1}{|c|}{ Items } & Classification Error \\
\hline Training Error (2957 cases, mixed) & $1.18 \%$ \\
\hline Testing Error (401 cases, Corridor) & $0.50 \%$ \\
\hline Testing Error (746 cases, Lecture Room) & $2.82 \%$ \\
\hline Testing Error (1809 cases, Office Room) & $<0.01 \%$ \\
\hline
\end{tabular}

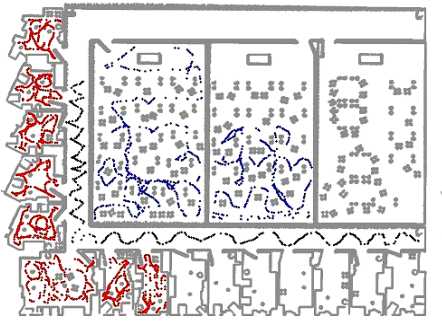

(a)

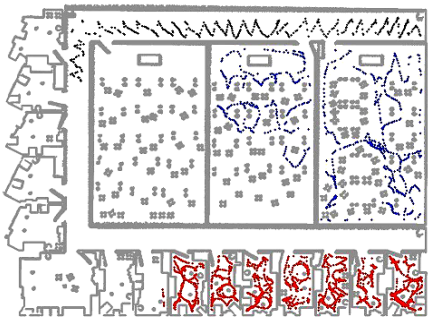

(b)
Fig. 3. (a) Training data and (b) Testing data in randomly opened doors scenario. The grey points depict the background map as a reference. Red, black and blue points are observer positions which are labeled as in office room, corridor and lecture room environment respectively (training data set is manually labeled and testing data set is labeled by classifier).

To evaluate the effect of door status, data set DS3, which is taken from the same map with fully opened doors, is employed to establish a reference group. Accordingly, the performance of the classifiers is shown in TABLE III.

Comparison between TABLE II and TABLE III indicates that the presence of opened doors leads to poor performance of the classifier. 
TABLE III

PERFORMANCE OF CLASSIFIER ON FULLY OPENED DOORS SCENARIO

\begin{tabular}{|l|c|}
\hline \multicolumn{1}{|c|}{ Items } & Classification Error \\
\hline Training Error (4718 cases, mixed) & $2.16 \%$ \\
\hline Testing Error (817 cases, Corridor) & $0.61 \%$ \\
\hline Testing Error (1154 cases, Lecture Room) & $6.76 \%$ \\
\hline Testing Error (2746 cases, Office Room) & $<0.01 \%$ \\
\hline
\end{tabular}

\section{Rectangle Filter}

Rectangle filter is proposed as a preprocessing method to discriminate inliers and outliers perceived in a single observation. It is utilized based on the hypothesis that most of the inliers lie in a rectangle, and the area of the rectangle is restricted by the area of polygonal approximation of all data points (Section IV.B). The results of rectangle filter are shown in Fig. 4. As can be seen, it provides a reasonable gross discrimination of inliers and outliers, and it is a conservative approach. This is evident especially in lecture room scenario (Fig. 4(b)).

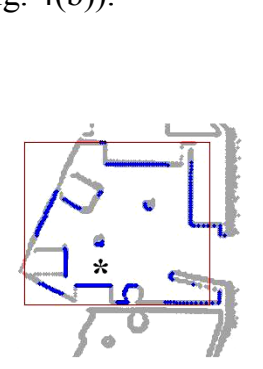

(a)

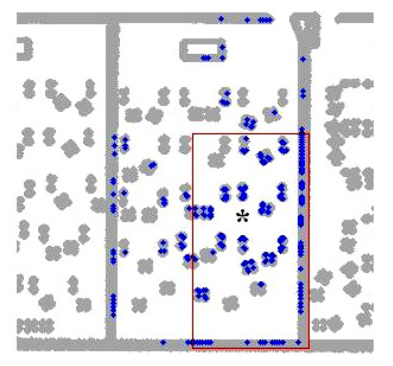

(b)

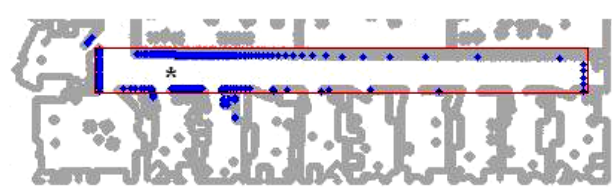

(c)

Fig. 4. Performance of rectangle filter: The observer marked as a star is in (a) office room, (b) lecture room and (c) corridor. The grey points depict the background map as a reference. The blue points are those detected by laser sensor. Points inside the red rectangle are considered to be inliers.

\section{E. Data Fusion}

This section concerns the semantic labels of perceived obstacle points in observations, rather than the observer's positions. The hierarchical relation of points is defined in the Venn diagram shown in Fig. 5, from which we derive equations (6) and (7) as the measure of the performance of classifier.

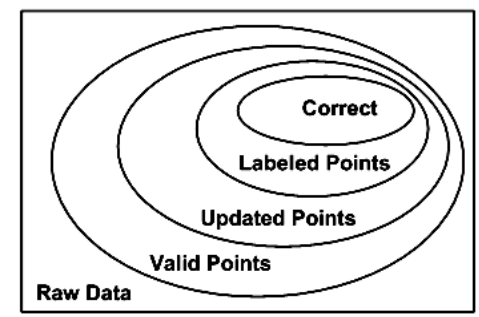

Fig. 5. Venn diagram of points. Valid Points are those within the range of LRF. Updated Points are those recognized as inliers by the rectangle filter. Labeled Points are those have more than $90 \%$ confidence of belonging to a certain class. Correct Points are those correctly labeled.
Following definitions have been used in the rest of the paper.

$$
\begin{aligned}
& \text { accuracy }=\frac{\text { correctly labeled points }}{\text { labeled points }} \times 100 \% \\
& \text { labeled rate }=\frac{\text { labeled points }}{\text { valid points }} \times 100 \%
\end{aligned}
$$

Data set DS2 with randomly opened door scenarios is used to build the semantic grid maps with outlier removal algorithm. The results are shown in TABLE IV.

TABLE IV

PERFORMANCE OF CLASSIFIER ON RANDOMLY OPENED DOORS SCENARIO

\begin{tabular}{|l|c|c|}
\hline \multicolumn{1}{|c|}{ On office room environment, 201 cases } \\
\hline \multicolumn{1}{|c|}{ Items } & Direct Data Fusion & Selective Data Fusion \\
\hline Labeled rate & $>99.99 \%$ & $84.07 \%$ \\
\hline Accuracy & $92.63 \%$ & $99.55 \%$ \\
\hline
\end{tabular}

\begin{tabular}{|l|c|c|}
\hline \multicolumn{3}{|c|}{ On lecture room environment, 201 cases } \\
\hline \multicolumn{1}{|c|}{ Items } & Direct Data Fusion & Selective Data Fusion \\
\hline Labeled rate & $86.25 \%$ & $64.25 \%$ \\
\hline Accuracy & $97.92 \%$ & $99.80 \%$ \\
\hline
\end{tabular}

\begin{tabular}{|l|c|c|}
\hline \multicolumn{3}{|c|}{ On corridor environment, 201 cases } \\
\hline \multicolumn{1}{|c|}{ Items } & Direct Data Fusion & Selective Data Fusion \\
\hline Labeled rate & $84.98 \%$ & $80.72 \%$ \\
\hline Accuracy & $94.58 \%$ & $97.18 \%$ \\
\hline
\end{tabular}

In TABLE IV, "selective data fusion" refers to utilization of independent opinion pool with rectangle fit outlier removal whereas "direct data fusion" does not utilize the outlier removal strategy. It can be observed from the table that in all cases, labeling accuracy rises at the cost of lower labeled rate with the application of rectangle filters.

As a comparison, the same data fusion approaches were applied on data set DS3 using the same map with fully opened doors. Results shown in TABLE $\mathrm{V}$ indicate the same trend as randomly opened door scenario, however with lower accuracies when compared with Table IV.

TABLE V

PERFORMANCE OF CLASSIFIER ON FULLY OPENED DOORS SCENARIO

\begin{tabular}{|l|c|c|}
\hline \multicolumn{3}{|c|}{ On office room environment, 204 cases } \\
\hline \multicolumn{1}{|c|}{ Items } & Direct Data Fusion & Selective Data Fusion \\
\hline Labeled rate & $>99.99 \%$ & $82.70 \%$ \\
\hline Accuracy & $89.60 \%$ & $99.01 \%$ \\
\hline
\end{tabular}

\begin{tabular}{|l|c|c|}
\hline \multicolumn{3}{|c|}{ On lecture room environment, 193 cases } \\
\hline \multicolumn{1}{|c|}{ Items } & Direct Data Fusion & Selective Data Fusion \\
\hline Labeled rate & $76.88 \%$ & $57.60 \%$ \\
\hline Accuracy & $95.07 \%$ & $96.24 \%$ \\
\hline
\end{tabular}

\begin{tabular}{|l|c|c|}
\hline \multicolumn{3}{|c|}{ On corridor environment, 205 cases } \\
\hline \multicolumn{1}{|c|}{ Items } & Direct Data Fusion & Selective Data Fusion \\
\hline Labeled rate & $74.90 \%$ & $70.53 \%$ \\
\hline Accuracy & $93.11 \%$ & $96.95 \%$ \\
\hline
\end{tabular}

An application of semantic grid map building is shown in Fig. 6, where we consider a robot building a semantic grid map while it moving along a trajectory. Assuming complete knowledge of robot localization, the semantic map was 
updated as shown in Fig. 7 (a) and (b). The figures show that the semantic map built using the selective data fusion strategy is more accurate than the direct data fusion strategy.

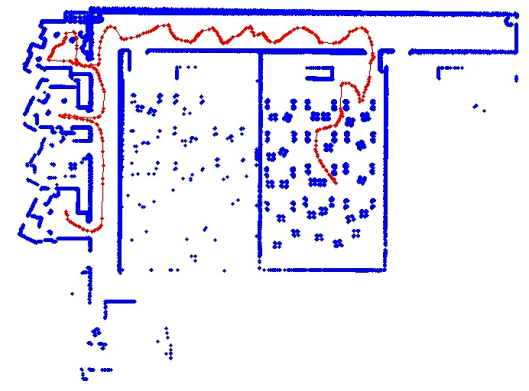

Fig. 6. Trajectory of a robot. Blue points are points detected by LRF; red points are observer's positions, which are points on the robot's trajectory.

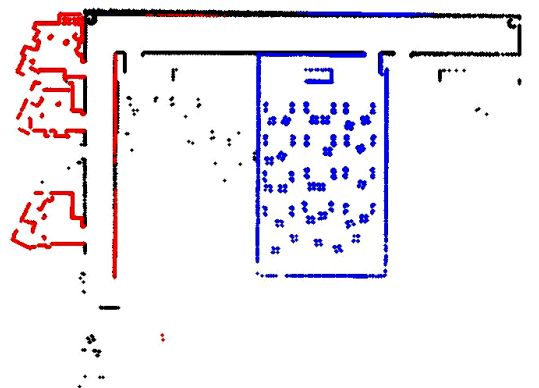

(a)

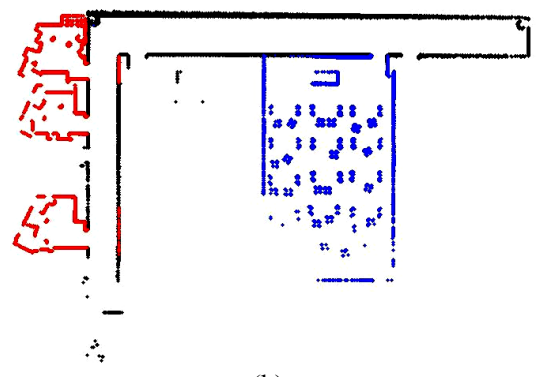

(b)

Fig. 7. Labeled map (a) with no filter applied (direct data fusion) and (b) with rectangle filter applied (selective data fusion). The red, black and blue points are detected points and finally labeled as belonging to office room, corridor, and lecture room environment respectively.

\section{CONCLUSIONS}

In this paper, we have presented an approach to classify the environment perceived by a robot equipped with LRF into three semantic classes, and then applied the classification outputs together with partial understanding of the geometric structure to semantically label scenes, and finally generated a semantic grid map. In the process, L2-regularized logistic regression was used as a multi-class classifier, independent opinion pool algorithm was adopted as data fusion strategy and a rectangle filter was implemented to discriminate inliers and outliers in a single scan. Rather than the conventional approach of labeling robot location, the methodology labels data points referring to the environment. The experimental results are appealing.

Currently, we are in the process of testing the algorithm on a real data on a real robot.

\section{ACKNOWLEDGMENT}

This work is supported by the ARC Centre of Excellence programme, funded by the Australian Research Council (ARC) and the New South Wales State Government.

\section{REFERENCES}

[1] WX. Shi, J. Samarabandu, "Investigating the Performance of Corridor and Door Detection Algorithms in Different Environments",

International Conference on Information and Automation, pp. 206 - 211, 2006

[2] P. Buschka, A. Saffiotti, "A virtual sensor for room detection", IEEE/RSJ International Conference on Intelligent Robots and Systems, vol. 1, pp. 637 - 642, 2002

[3] A. Poncela, C. Urdiales, B. Fernandez-Espejo, F.Sandoval, "Place characterization for navigation via behaviour merging for an autonomous mobile robot", The 14th IEEE Mediterranean Electrotechnical Conference, pp. 350 - 355, 2008

[4] A.Tapus, G. Ramel, L. Dobler, R. Siegwart, "Topology learning and recognition using Bayesian programming for mobile robot navigation", IEEE/RSJ International Conference on Intelligent Robots and Systems, vol. 4, pp. 3139 - 3144, 2004

[5] P. Viswanathan, D. Meger, T. Southey, J.J. Little, A.K. Mackworth "Automated Spatial-Semantic Modeling with Applications to Place Labeling and Informed Search", Canadian Conference on Computer and Robot Vision, pp. 284 - 291, 2009

[6] O.M. Mozos, C. Stachniss, W. Burgard, "Supervised Learning of Places from Range Data using AdaBoost", Proceedings of the IEEE

International Conference on Robotics and Automation. pp. 1730 - 1735, 2005

[7] P. Sousa, R. Araiijo, U. Nunes, "Real-Time Labeling of Places using Support Vector Machines", IEEE International Symposium on Industrial Electronics, pp. 2022 - 2027, 2007

[8] T. Mitchell. (2005). Generative and Discriminative Classifiers: Naive Bayes and Logistic Regression [Online]. Available: http://www.cs.cmu.edu/ tom/mlbook/NBayesLogReg.pdf

[9] O.M. Mozos, Semantic Place Labeling [Online]. Available: http://www.informatik.uni-freiburg.de/ omartine/place_data_sets.html File: fr52.tgz

[10] A.Y. Ng, "Feature selection, L1 vs. L2 regularization, and rotational invariance", International Conference on Machine Learning, 2004

[11] R.E. Fan, K.W. Chang, C.J. Hsieh, X.R. Wang, and C.J. Lin. "LIBLINEAR: A library for large linear classification", Journal of Machine Learning Research, vol. 9, pp. 1871-1874, 2008

[12] Y. Freund, R. Schapire, N. Abe, "A Short Introduction to Boosting", Journal of Japanese Society for Artificial Intelligence, vol. 14(5), pp. 771-780, 1999

[13] C.J.C. Burges, "A Tutorial on Support Vector Machines for Pattern Recognition", Data Mining and Knowledge Discovery, vol. 2(2), pp. 147, 1998

[14] A. Pronobis, O. M. Mozos, B. Caputo, and P. Jensfelt, "Multi-modal semantic place classification", International Journal of Robotics Research, vol. 29, pp. 298-320, 2010

[15] O. M. Mozos, "Semantic Place Labeling with Mobile Robots", PhD thesis, Dept. of Computer Science, University of Freiburg, 2008.

[16] T.Mitchell, Machine Learning, New York : McGraw-Hill, 1997, pp. 2-4.

[17] J.O. Berger, Statistical decision theory and Bayesian analysis, 2nd ed., New York: Springer-Verlag, 1985, pp. 272-277

[18] J.R. Raol, Multi-Sensor Data Fusion: Theory and Practice, Boca Raton: CRC Press, 2010, pp. 60-61

[19] R. Siegwart, I.R. Nourbakhsh, Introduction to autonomous mobile robots, Cambridge: MIT Press, 2004, pp. 200-212

[20] D. Anguelov, D. Koller, E. Parker, S. Thrun, "Detecting and modeling doors with mobile robots", IEEE International Conference on Robotics and Automation, vol. 4, pp. 3777 - 3784, 2004

[21] P. Carinena, C.V. Regueiro, A. Otero, A.J. Bugarin, S. Barro, "Landmark detection in mobile robotics using fuzzy temporal rules", IEEE Transactions on Fuzzy Systems, vol. 12(4), pp. 423 - 435, 2004 